\title{
Id proteins sustain Hes1 expression to maintain neural stem cells
}

Ge Bai ${ }^{1}$, Nengyin Sheng ${ }^{1}$, Zhihui Xie ${ }^{1}$, Wei Bian ${ }^{1}$, Yoshifumi Yokota ${ }^{2}$, Robert Benezra ${ }^{3}$, Ryoichiro Kageyama ${ }^{4}$, Francois Guillemot ${ }^{5}$, Naihe Jing ${ }^{1}$

${ }^{1}$ Laboratory of Molecular Cell Biology, Key Laboratory of Stem Cell Biology, Institute of Biochemistry and Cell Biology, Shanghai Institutes for Biological Sciences, Chinese Academy of Sciences, China, ${ }^{2}$ Department of Molecular Genetics, School of Medicine, University of Fukui, Japan, ${ }^{3}$ Department of Cancer Biology and Genetics, Memorial Sloan-Kettering Cancer Center, USA, ${ }^{4}$ Institute for Virus Research, Kyoto University, Japan, ${ }^{5}$ Division of Molecular Neurobiology, National Institute for Medical Research, UK

Negative bHLH transcription factor Hes1 can inhibit neural stem cells (NSCs) from precocious neurogenesis through repressing proneural gene expression; therefore, sustenance of Hes1 expression is crucial for NSC pool maintenance. Here, we find that Ids, the dominant-negative regulators of proneural proteins, are expressed prior to proneural genes and share an overlapping expression pattern with Hes1 in the early neural tube of chick embryos. Overexpression of Id2 in the chick hindbrain upregulates Hes1 expression and inhibits proneural gene expression and neuronal differentiation. By contrast, Hes1 expression decreases, proneural gene expression expands and neurogenesis occurs precociously in Id1;Id3 double knockout mice and in Id1-3 RNAi-electroporated chick embryos. Mechanistic studies show that Id proteins interact directly with Hes1 and release the negative feedback autoregulation of Hes1 without interfering with its ability to affect other target genes. These results indicate that Id proteins participate in NSC maintenance through sustaining Hes1 expression in early embryos.

Keywords: neural stem cell, maintenance, bHLH, proneural gene

Cell Research (2008) 18:s19. doi: 10.1038/cr.2008.109; published online 4 August 2008

Correspondence: Naihe Jing

E-mail: njing@sibs.ac.cn

Dr Naihe Jing, Professor \& Deputy Director of Institute of Biochemistry and Cell Biology, Shanghai Institutes for Biological Sciences, Chinese Academy of Sciences. The main research interest of Dr. Jing is the molecular mechanism of neural development and embryonic stem cell neural differentiation. Using mouse embryonic carcinoma P19 cells and mouse embryonic stem cells, Dr Jing's group studies the involvement of BMP and FGF signaling pathways in pluripotent stem cell neural determination. Dr Jing's group also found that Id proteins sustain Hes 1 gene expression to maintain neural stem cell pool in early chick and mouse embryos. 